\title{
Treatment of Obstructive Sleep Apnea and Hipoapnea Syndrome with oral appliances
}

\author{
Marco Antonio de Oliveira Almeida ${ }^{1}$, Andressa \\ Otranto de Britto Teixeira ${ }^{2}$, Livia Saladini Vieira ${ }^{3}$, \\ Cátia Cardoso Abdo Quintãó
}

Keywords: Apnea. Snoring. OSAHS. Oral Appliances.

\section{Summary}

\begin{abstract}
$\mathrm{O}$
bstructive sleep apnea and hipoapnea syndrome (OSAHS) is a disorder that affects about $4 \%$ of the adult population, and besides the social problems associated to snoring and extreme day time sleepiness, it is preoccupying since it may cause pulmonary hypertension and cardiac failure. Review and discussion - Through a literature review, we discuss the use of oral appliances to treat this condition, in regards of therapy effectiveness and limitations, main clinical symptoms, major occlusal side effects, rate of improvement and patient satisfaction. Conclusions - We concluded that the use of oral appliances should be a first choice treatment for mild to moderate OSAHS, being dental, joint and muscular discomforts, hypersalivation and xerostomia, the most frequent clinical symptoms, with light occlusal side effects that normally do not bother the patients, with a good degree of improvement and high satisfaction index.
\end{abstract}

${ }^{1} \mathrm{PhD}$ from the North Carolina University, Professor of Orthodontics at UERJ.

${ }^{2}$ Dental Surgeon, specialist in Orthodontics by ABO /JF, MSc Dentistry Student - Concentration Area: Orthodontics at UERJ

${ }^{3}$ Dental Surgeon, Orthodontics Specialization Student at UERJ

${ }^{4} \mathrm{PhD}$ in Orthodontics at UFRJ, Associated Professor of Orthodontics at UERJ. State University of Rio de Janeiro.

Mail address: Marco Antonio de Oliveira Almeida - Boulevard 28 de Setembro $1572^{\circ}$ andar Vila Isabel Rio de Janeiro RJ 20551-030.

Paper submitted to the ABORL-CCF SGP (Management Publications System) on September 9th, 2005 and accepted for publication on August 25 th, 2006. Cod. 1162. 


\section{INTRODUCTION}

Since 1985, Medicine and Dentistry have increased its focus on sleep respiratory disorders. Although there is a large number of disorders that may be included in this category, obstructive sleep apnea and the choices of its possible intra-oral treatments, have received more attention.

Obstructive sleep apnea and hypopnea syndrome (OSAH) is defined by the apnea/hypopnea index (AHI) per hour of at least five events per hour, added to clinical symptoms, being loud snoring and excessive daytime sleepiness, the most important ones. ${ }^{1,2}$

This syndrome affects as many as $4 \%$ of the adult population, being more predominant in men, increasing from the 5 th life decade onwards ${ }^{3,4}$. Patients with OSAH have significantly more hypertension, ischemic heart disease and brain vascular disease than individuals without the syndrome. Mortality related to obstructive sleep apnea has increased significantly, when the AHI $>20$ events per hour ${ }^{5}$.

Some important anatomic features observed radiographically in patients with OSAH include: narrow mandible arch; maxillary and mandibular retrognathism; increased lower facial height; lower and more anterior position of the hyoid bone; reduced pharyngeal area; increased cranio-cervical angle; decreased distance between the base of the tongue and the posterior pharyngeal wall; hypertrophied tonsils and adenoids ${ }^{6-10}$, over-erupted maxillary and mandibular dentition and enlarged tongue ${ }^{6,7}$.

OSAH treatment goal is to restore normal breathing during sleep, and therefore, ending excessive daytime sleepiness, neuropsychological and cardiovascular alterations; and at the same time it should offer the patient good quality of life, without offering side effects or risks ${ }^{11}$.

Treatment options for OSAH include sleep hygiene, that is to say, avoidance of alcohol and other drugs; adequate body position and weight loss ${ }^{11}$, surgical procedures, such as partial glossectomy, uvulopalatopharyngoplasty, maxillary and mandibular advancement procedures, and also clinical treatments, such as nasal CPAP and intra-oral devices ${ }^{12}$.

The most commonly used clinical treatment includes the continuous positive air pressure procedure (CPAP) applied through a device that generates and directs a continuous air flow ( 40 to $601 / \mathrm{min}$.), through a flexible tube to a nasal mask tightly strapped to the patient's face. 3,11. According to Sullivan et al. ${ }^{5}$, although the treatment of obstructive sleep apnea with CPAP is highly effective, it is little tolerated by approximately $36 \%$ of patients, specially by those with medium severity.

There has been a recent increased interest on the principle of treatment with intra-oral devices, because they are a simple and non-invasive option, different de- vices have been developed. ${ }^{5}$. The devices approved by the Food and Drug Administration (FDA) are the tongue retainers and mandibular protruding devices, being the most widely used options ${ }^{2}$.

However, some questions need to be addressed in order to make this therapy into an efficient and safe alternative for treating such respiratory disorders. These questions are related to the correct indication regarding the severity of the condition ${ }^{5}$, that is to say, which degrees of apnea could be treated with this therapeutic option, patient's comfort when using the intra-oral device ${ }^{13-15}$, user's and room mate's evaluation regarding the improvement of the clinical condition ${ }^{14}$ and side effects.

The goal of this paper is based on the review of recent literature, and to check the following:

1 - Mandibular protruding devices efficacy for treating obstructive sleep apnea.

2 - Main clinical symptoms informed by patients.

3 - Main occlusal side effects produced by mandibular protruding devices.

4 - Patients' collaboration level and satisfaction rate regarding the treatment.

\section{DISCUSSION}

Regarding the efficacy of intra-oral devices, several authors state in their studies ${ }^{16-19}$ that they represent a good alternative for treating snoring and OSAH, due to a reduced cost and their relatively comfortable use, being more accepted by patients. Although there are conflicting doubts on the efficacy of intra-oral devices for treating snoring and $\mathrm{OSAH}$, its use in the treatment of medium severity apnea has received great attention and acceptability. ${ }^{8}$

A literature review done by Warunek (2004)10 showed that since 1985, approximately 150 articles have been published describing different devices and its efficacy in treating OSAH.

The mechanics of mandibular protruding devices aims to increase upper airway volume ${ }^{2}$, having as a result mandibular advancement, which places the tongue away from the posterior pharyngeal wall, lowering this bone and moving the tongue away from the soft palate ${ }^{20}$, altering the position of the hyoid bone and therefore, modifying the space of the hypopharyngeal airway. ${ }^{3}$

Regarding its effectiveness, Walker-Engström et al. $(2002)^{4}$ managed to get at least a 50\% improvement in the apnea - hypopnea index (AHI) in $81 \%$ of patients, and normalization in $63 \%$ of patients who used intra-oral devices which promoted mandibular advancement of 50\% of protrusion maximum capacity. Using polysomnography, Neill et al. (2002) ${ }^{14}$ evaluated the use of intra-oral devices of mandibular protrusion and concluded that it was a complete success in $21.1 \%$ of patients, partial success in $52.6 \%$ and a failure in $26.3 \%$, with an average treatment period of 6.5 weeks. According to the study done by Fritsch et al. 
$(2001)^{13}$, a respiratory improvement was confirmed with the device, when all patients showed a persistent relief of symptoms, 12 to 30 weeks after its use.

In the study done by Pancer, Al-Faifi \& Hoffstein (1999)20, they observed a significant reduction in all rates related to respiratory disorders and the subjective assessment of room mates revealed a striking improvement in snoring (96\% were evaluated as "loud snoring" frequently before using the device and only $2 \%$ using the device). The objective analysis showed that of the 75 patients, 38 were cured (normalization of AH index), 31 improved $\mathrm{AH}$ index without normalization, three were only snorers, and three had a worse $\mathrm{AH}$ index with the device. According to Katto et al. (2000) ${ }^{17}$, among the patients who had mandibular advancement of at least $6 \mathrm{~mm}$, there was an improvement of their condition with normalization in $65 \%$. They also reported that patients who normalized their indexes were the less obese of the study and suggested that obese patients should not be suitable candidates to therapy with oral devices. In the study done by Ferguson et al. (1997) ${ }^{16}$, when compared to the non-adjustable mandibular repositioning device, the adjustable mandibular protruding device, was associated with a higher success of treatment ( $55 \%$ vs. 48\%), and fewer failures (5\% vs. 24\%). For Clark et al. (1993) ${ }^{21}$, the follow-up makes clear that when the protrusive position was lower than $75 \%$ of maximum protrusion reached by the patient (normally < $5 \mathrm{~mm}$ ) the advancement therapy did not work, and they stated that for those patients who do not tolerate such a degree of advancement, this would not be the indicated therapy.

For Fransson et al. $(2004)^{22}$, the treatment with mandibular protruding devices has several advantages and can be considered as first choice treatment for a large group of patients, including patients with severe OSAH, if an optimum advancement quantity could be used. In our sample, more than $80 \%$ of patients with OSAH normalized their values of oxygen saturation (with an improvement of $50 \%$ or more), and $90 \%$ reported a subjective reduction of snoring and apnea (50\% reduction or more).

Regarding clinical symptoms, dental or mouth discomfort were the most frequently symptoms reported by patients in all samples. In the work done by Neill et al. $(2002)^{14}, 26 \%$ of patients experienced some pain in the mouth and $42 \%$ reported sore teeth and gums. Pancer, AlFaifi \& Hoffstein (1999) ${ }^{20}$ reported dental discomfort some times or with frequency in more than $32 \%$ of patients. According to Clark, Sohn \& Hong $(2000)^{3}, 38 \%$ of patients reported suffering from toothache. For O'Sullivan $(1995)^{5}$, the main effect reported was medium discomfort in the dental arcs when waking up, with reduction of the symptom in three weeks, in most cases. Besides discomfort, joint and masticatory musculature pain was also reported, found in different levels in the works by $2,4,8,9,12,22$. Other frequent clinical symptoms were excessive salivation ${ }^{8,13}$ due to the new tongue position and to the presence of the device inside the mouth ${ }^{2,3,8,9,12,13,14,20}$, and xerostomia ${ }^{3,5}$. Fransson et al. (2004) 22 and Bondemark \& Lindman (2000) 9 also reported the frequency reduction in headache in patients of their samples.

Among the frequently observed occlusal alterations, there are overjet, overbite reduction, lower incisive proclin ation $^{9,10,13,18,21,22}$, and the establishment of lateral open bite. 22 According to Fransson et al. (2002) ${ }^{23}$, a small overjet is a limiting factor to be considered in the treatment with this type of device.

According to Robertson (2001) ${ }^{24}$, the main changes observed are due to the vertical repositioning of the mandibular condyle in the glenoid cavity. These alterations may become visible within 6 months. Dental changes start happening later on, and they will be significant at the 30 month follow-up. Bondemark \& Lindman (2000)9 carried out a two year follow-up work and they observed a significant decrease in overjet and overbite, and significant changes in molar relation, indicating a more mesial sagittal relation and/or changes in the incisive inclination.

Some authors ${ }^{25-27}$ correlate the observed occlusal changes with the type of initial occlusion in patients using mandibular protruding devices for OSAH treatment. Almeida et al. (2006) $)^{25}$ and Almeida et al. (2006) ${ }^{26}$ carried out some long term assessment works, 4.7 years average. Five orthodontists made a visual comparison of the dental arcades plaster molds of 70 patients, and 71 patients had their cephalometric x-rays analyzed. In the analysis of the models, the authors verified that changes occur in $85.7 \%$ of cases, being favorable or unfavorable depending on the initial mal-occlusion. On the other hand, on the cephalometric assessment, they observed a decrease of overjet and overbite, changes in the incisive and molar teeth position and consequent alteration in the mandibular position, also varying depending on the initial occlusion. Marklund (2006) ${ }^{27}$ confirmed this correlation between the observed changes and initial occlusion on their work with 187 patients, during an average follow-up period of 5.4 years.

The assessment of the patient compliance should be done in order to be able to indicate the treatment with mandibular protruding device in an effective manner, since this appears to be the main difficulty for treatment with CPAP. Compliance depends on the comfort level obtained by patient during the use of the devices and their own assessment regarding the result of the therapy. All the studies show a high level of compliance in using the device, and always in a higher level than the use of CPAP when they are compared ${ }^{2-5,14,16,20,22}$. According to Almeida (2002) ${ }^{2}$ and Ferguson et al. (1997) ${ }^{16}$ patients have a significant preference for the use of mandibular protruding devices rather than the use of CPAP, even those for whom CPAP efficacy 
was better than intra-oral devices. Walker-Engströn et al. $(2002)^{4}$ found a compliance level of $82 \%$ after one year and $62 \%$ after four years. Fransson et al. (2004)22 found an $84 \%$ compliance rate on the two year follow-up. Clark, Sohn \& Hong $(2000)^{3}$ reported that with the long term use (three or more years), compliance was higher than 51\%, whereas Pancer, Al-Faifi \& Hoffstein (1999) ${ }^{20}$ stated that 350 days after device fitting, $86 \%$ of patients still were using it. According to Neill et al. (2002) $)^{14}$ the device was used by $53 \%$ of patients every night, by $26 \%$ more than three night a week, by $21 \%$ less than that. A high satisfaction rate was reported by articles assessing this item. ${ }^{4,9,20}$.

\section{CONCLUSIONS}

From the bibliography review focused on answering the main doubts on the use of intra-oral devices for treating $\mathrm{OSAH}$, we can conclude the following:

1- Intra-oral devices for mandibular advancement can be considered as first choice treatment for patients with medium to moderate OSAH, because the devices show a significant condition improvement and a greater acceptability from patients when compared to CPAP. These types of devices can be tried in patients with severe OSAH when they are intolerant to treatment with CPAP.

2- $\quad$ The main clinical symptoms informed by patients are dental, joint and muscular discomfort, hyper salivation and xerostomia, reduction in headache frequency and improvement of snoring (frequency and intensity).

3- The main occlusal side effects found were overjet reduction, overbite reduction, lower incisive proclination, establishment of lateral open bite. However, most of the time without causing great discomfort to patients.

4- Treatment with mandibular protruding devices show a higher compliance level than

CPAP, even in patients who had a higher efficacy with CPAP than with intra-oral devices.

Regarding patient's satisfaction with treatment, it was observed a high rate in all the studies

that included this item in their assessments.

\section{REFERENCES}

1. Pignatari SSN, Pereira FC, Avelino MAG, Fujita RR. Noções gerais sobre a síndrome da apnéia e da hipopnéia obstrutiva do sono em crianças e o papel da polissonografia. In: Campos CAH, Costa HOO (eds.). Tratado de Otorrinolaringologia. 1aㅡ ed. São Paulo: Editora Roca; 2002. p. 577-9.

2. Almeida FR. Aparelhos intrabucais para o tratamento do ronco e síndrome da apnéia e hipopnéia obstrutivas do sono. In: Campos $\mathrm{CAH}$, Costa HOO (eds.). Tratado de Otorrinolaringologia, 1a ed. São Paulo: Editora Roca; 2002. p. 594-1.

3. Clark GT, Sohn JW, Hong CN. Treating obstructive sleep apnea and snoring: assessment of an anterior mandibular positioning device. J Am Dent Assoc 2000;131(6):765-1.
4. Walker-Engström ML, Tegelberg A, Wilhelmsson B, Ringqvist I. Four-year follow-up of treatment with dental appliance or uvupalatopharyngoplasty in patients with obstructive sleep apnea. Chest 2002;121(3):739-6.

5. O'Sullivan R, Hillman D, Mateljan R, Pantin C, Finucane K. Mandibular advancement splint: an appliance to treat snoring and obstructive sleep apnea. Am J Respir Crit Care Med 1995;151:194-8.

6. Wong ML, Sandham A, Ang PK, Wong DC, Tan WC, Huggare J. Craniofacial morphology, head posture, and nasal respiratory resistance in obstructive sleep apnoea: an inter-ethnic comparison. Eur J Orthod 2005;27(1):91-7.

7. Ang PK, Sandhan A, Tan WC. Craniofacial morphology and head posture in Chinese subjects with obstructive sleep apnea. Semin Orthod 2004;10(1):90-6.

8. Bondemark L. Does 2 years nocturnal treatment with a mandibular advancement splint in adult patients with snoring and OSAS cause a change in the posture of the mandible? Am J Orthod Dentofacial Orthop 1999;116(12):621-8.

9. Bondemark K, Lindman R. Craniomandibular status and function in patients with habitual snoring and obstructive sleep apnea after nocturnal treatment with a mandibular advancement splint: a 2-year follow-up. Eur J Orthod 2000;22:53-60.

10. Warunek SP. Oral appliance therapy in sleep apnea syndroms: a review. Sem Orthod 2004;10(1):73-89.

11. Bittencourt LRA. Tratamento clínico da síndrome da apnéia e hipopnéia obstrutiva do sono. In: Campos CAH, Costa HOO (eds.): Tratado

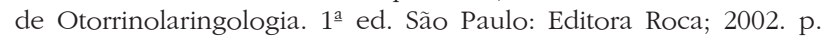
584-3.

12. Fujita RR, Moysés MG, Vuono IM. Ronco e apnéia do sono. In: Campos CAH, Costa HOO (eds.). Tratado de Otorrinolaringologia. 1aㅡ ed. São Paulo: Editora Roca; 2002. p. 637-3.

13. Fritsch KM, Iseli A, Russi EW, Bloch KE. Side effects of mandibular advancement devices for sleep apnea treatment. Am J Respir Crit Care Med 2001;164:813-8.

14. Neill A, Whyman R, Bannan S, Joffrey O, Campbell A. Mandibular advancement splint improves indices of obstructive sleep apnea and snoring but side effects are common. NZ Med J 2002;115:289-2.

15. Pantin CC, Hillman DR, Tennant M: Dental side effects of an oral device to treat snoring and obstructive sleep apnea. Sleep 1999;22(2):23740 .

16. Ferguson KA, Ono T, Lowe AA, Al-Majed S, Love LL, Fleetham JA A short term controlled trial of an adjustable oral appliance for the treatment mild to moderate obstructive sleep apnea. Thorax 1997;52:362-8.

17. Katto J, Isono S, Tanaka A, Watanable T, Araki D, Tanzawa $\mathrm{H}$, Nishino T. Dose-dependent effects on mandibular advancement on pharyngeal mechanics and nocturnal oxygenation in patients with sleep-disordered breathing. Chest 2000;117(4):1065-2.

18. Metha A, Qian J, Petoez P, Darendeliler MA, Cistulli PA. A randomized controlled study of a mandibular advancement splint for obstructive sleep apnea. Am J Respir Crit Care Med 2001;163:1457-1.

19. Randerath WJ, Heise M, Hinz R, Ruehle K-H. An individually adjustable oral appliance vs continuous positive airway pressure in mild-tomoderate obstructive sleep apnea syndrome. Chest 2002;122(2):5695.

20. Pancer J, Al-Faifi S, Hoffstein V. Evaluation of variable mandibular advancement appliance for treatment of snoring and sleep apnea. Chest 1999;116(6):1511-8.

21. Clark GT, Arand D, Chung E, Tong D. Effect of anterior mandibular positioning on obstructive sleep apnea. Am Rev Respir Dis 1993;147:624-9

22. Fransson AMC, Tegelberg A, Johansson A, Wenneberg B. Influence on the masticatory system in treatment of obstructive sleep apnea and snoring with a mandibular protruding device: A 2-year followup. Am J Orthod Dentofacial Orthop 2004;126:687-3.

23. Fransson AMC, Tegelberg A, Svenson BAH, Lennartsson B, Isacsson G. Influence of mandibular protruding device on airway passages and dentofacial characteristics in obstructive sleep apnea and snoring. 
Am J Orthod Dentofacial Orthop 2002;122(10):371-9.

24. Robertson CJ. Dental and skeletal changes associated with long-term mandibular advancement. Sleep 2001;24(5):531-7.

25. Almeida FR, Lowe AA, Sung JO, Tsuiki S, Otsuka R. Long-term sequellae of oral appliance therapy in obstructive sleep apnea patients: Part 1. Cephalometric analysis. Am J Orthod Dentofacial Orthop 2006,129(2):195-4.
26. Almeida FR, Lowe AA, Otsuka R, Fastlicht S, Farbood M, Tsuiki S: Long-term sequellae of oral appliance therapy in obstructive sleep apnea patients: Part 2. Study-model analysis. Am J Orthod Dentofacial Orthop 2006;129(2):205-3.

27. Marklund M: Predictors of long-term orthodontic side effects from mandibular advancement devices in patients with snoring and obstructive sleep apnea. Am J Orthod Dentofacial Orthop 2006;129(2):214-1. 